\title{
ATUAÇÃO DO ENFERMEIRO NAS ESCOLAS PARA A PREVENÇÃO DA GRAVIDEZ NA ADOLESCÊNCIA
}

\section{ARTIGO DE REVISÃO}

CAMPOS, Rubilanny de Souza Ferreira ${ }^{1}$

SILVA, Claudia Lucrécia de Matos ${ }^{2}$

CAMPOS, Rubilanny de Souza Ferreira. SILVA, Claudia Lucrécia de Matos. Atuação do enfermeiro nas escolas para a prevenção da gravidez na adolescência. Revista Científica Multidisciplinar Núcleo do Conhecimento. Ano 05, Ed. 10, Vol. 18, pp. 107-118. Outubro de 2020. ISSN: 2448-0959, Link de acesso: https://www.nucleodoconhecimento.com.br/saude/prevencao-da-gravidez

\section{RESUMO}

A gravidez na adolescência é um problema de saúde pública relevante no Brasil, pois a gestação nessa faixa etária origina uma série de complicações a mais para a saúde dessas mães e seus filhos, além de ocasionar impactos na sociedade. Desta forma o presente trabalho tem por objetivo relatar, através de uma revisão da literatura, quais ações o enfermeiro da Atenção Básica $(A B)$ pode desenvolver em sua área de abrangência para prevenção da gravidez na adolescência. O estudo foi desenvolvido através de uma análise da literatura, no formato de revisão bibliográfica narrativa. Realizando uma busca avançada na plataforma Scientific Electronic Library Online (Scielo), utilizando os termos "gravidez and adolescência and enfermagem". Foram encontrados setenta e três artigos, destes apenas sete se enquadraram nos seguintes

\footnotetext{
${ }^{1}$ Acadêmica de Enfermagem.
}

2 Orientadora. Mestrado em andamento em Mestrado em Gestão de Cuidados da Saúde. Especialização em Ginecologia E Obstetricia. Especialização em PRM/PSUS - Preceptoria de Residência Médica no SUS/ Preceptoria no SUS. Especialização em Politicas Públicas e Programa de Saúde da Família. Graduação em Enfermagem. 
critérios de inclusão, ser de língua portuguesa, ter texto completo disponível, possuir publicação entre os anos de 2009 a 2019. Dos artigos encontrados apenas um abordava estratégias para prevenção da gravidez, este citou como método utilizado por alguns enfermeiros da atenção básica o trabalho com grupos, tendo em vista que facilitava a abordagem de temas, favorecia o desenvolvimento da autoestima e maturidade emocional dos adolescentes, cita ainda que quando a educação em saúde é trabalhada no modelo convencional se torna ineficaz para esse público, que as ações devem ser inovadoras, criativas, dinâmicas, motivadoras e instigantes, com o intuito de ampliar o vínculo dos adolescentes com os serviços de saúde. Os demais discorreram sobre outros pontos igualmente relevantes na atenção a gestação de adolescentes, bem como a percepção de médicos e enfermeiros sobre este grupo, avaliação da assistência as adolescentes na $A B$, durante o trabalho de parto e a percepção sobre o apoio social na maternidade. Embora os artigos analisados não abordem sobre a prevenção da gravidez na adolescência no ambiente escolar, requerendo uma pesquisa ampliada para tal, os resultados encontrados são de igual relevância e apontam para a necessidade de melhorias no atendimento ofertado pelos enfermeiros a esse público, tanto no ambiente hospitalar quanto na $A B$.

Palavras-Chave: Gravidez, adolescência, enfermagem.

\section{INTRODUÇÃO}

A adolescência é um período de grandes mudanças, tanto corporal, quanto psíquico e social, que predispõem a vulnerabilidade, principalmente naqueles com menos acesso aos bens sociais, fato que requer dos profissionais da saúde uma assistência ampla, capaz de acompanhar o desenvolvimento dessa população (FERNANDES; NARCHI, 2013).

Nesta fase da vida, em que ao invés de uma situação de equilíbrio, existe uma situação de crise e de mudanças, o evento de uma gravidez pode assumir uma dimensão imensa, levando as adolescentes a sofrerem vários efeitos sociais (BERETTA et. al., 2011). 
Esses impactos na relação social entre as adolescentes grávidas gera uma série de conflitos psicológicos, podendo desencadear depressões, ansiedades e perca da autoestima. No nível fisiológico, uma gestação na adolescência aumenta o risco para abortos, anemia e complicações no parto. Constata-se que há maior número de bebês prematuros, com peso inferior a 2500 gramas, necessitando de cuidados intensivos e maior dificuldade para execução do cuidado domiciliar, quando comparado com grávidas adultas (SOCIEDADE BRASILEIRA DE PEDIATRIA, 2019).

Segundo a Organização Mundial de Saúde - OMS (2011) "as complicações de gravidez e parto representam a principal causa de óbito em mulheres jovens com idade entre 15 e 19 anos nos países em desenvolvimento".

No Brasil em 2017, foi registrado 480.923 nascidos de mães com idade entre 10 e 19 anos, conforme o Sistema de Informações sobre Nascidos Vivos - SINASC, disponível no DATASUS. Dada a amplitude das questões relacionadas à vida e à saúde dos adolescentes, não é possível restringir as atividades do profissional enfermeiro apenas a cuidados clínico-educativos individuais (FERNANDES; NARCHI, 2013).

Tendo em vista que a atuação na Atenção Básica (AB) é caracterizada por ações individuais e coletivas, abrangendo a promoção, proteção, prevenção e recuperação da saúde da população com o intuito de ofertar uma atenção integral (BRASIL, 2012).

Torna-se imprescindível que o enfermeiro, membro da equipe de $A B$, desenvolva ações para a conscientização sobre a prevenção da gravidez na adolescência, atuando além dos muros da Unidade Básica de Saúde (UBS), ingressando nas escolas, pois são locais de fácil acesso a esse público.

A realização de atividades voltadas à atenção a saúde em outros locais do território, como escolas, creches e associações é uma característica do processo de trabalho das equipes da $A B$ previstos pela Política Nacional de Atenção Básica - PNAB (BRASIL, 2012). 
Desta forma o presente artigo tem por objetivo relatar, através de uma revisão da literatura, quais ações o enfermeiro pode desenvolver em sua área de abrangência para prevenção da gravidez na adolescência.

\section{DESENVOLVIMENTO}

Estudo desenvolvido através de uma análise da literatura, no formato de revisão bibliográfica narrativa. Foi realizada uma busca avançada na plataforma Scielo, utilizando os termos "gravidez and adolescência and enfermagem". Foram encontrados setenta e três artigos, destes apenas sete se enquadraram nos seguintes critérios de inclusão, ser de língua portuguesa, ter texto completo disponível, possuir publicação entre os anos de 2009 a 2019.

Dos artigos encontrados apenas um abordava estratégias para prevenção da gravidez, os demais discorreram sobre outros pontos igualmente relevantes na atenção a gestação de adolescentes, bem como a percepção de médicos e enfermeiros sobre este grupo, avaliação da assistência as adolescentes na Atenção Básica $(A B)$, durante o trabalho de parto e a percepção sobre o apoio social na maternidade.

O artigo de Braga et al. (2014), aponta presença ou falta de apoio social que as adolescentes recebem durante a gestação, através de entrevistas semi-estruturadas com vinte adolescentes numa Unidade Básica de Saúde (UBS) do interior de São Paulo, no ano de 2010.

Constatando que a principal fonte de apoio social no ambiente familiar se encontra na figura da mãe destas adolescentes, que os companheiros para algumas foi revelado como apoiador e para outras prevaleceu o sentimento de abandono por parte deles. Ainda no ambiente doméstico, os profissionais da saúde, enfermeiros, médicos, agentes comunitários de saúde e os professores foram apontados como fontes de apoio social. 
No contexto da maternidade, as adolescentes indicaram que perceberam quatro tipos de apoio, o emocional, informativo, instrumental e de reforço, os profissionais de saúde se enquadraram como ofertadores de apoio do tipo informativo, sugerindo, aconselhando, informando, demonstrando carinho, preocupação e atenção com as adolescentes.

Entretanto algumas apontaram falta de apoio por parte dos profissionais, tendo em vista que em diferentes momentos relataram dúvidas e desconhecimento sobre aspectos básicos dos cuidados com o bebê, revelando assim a necessidade de coordenar os serviços e a equipe de saúde para que possam atuar promovendo uma maternidade saudável.

Beretta et al. (2011) apresenta a experiência de um grupo de docentes da área materno-infantil, no curso de enfermagem, da Universidade Federal de São Carlos, que desenvolveram um projeto com o intuito de orientar a puérpera adolescente no período de um ano, realizando uma visita na maternidade e quatro em domicílio, respectivamente na primeira semana de vida do bebê, no primeiro, terceiro e sexto mês, este acompanhamento foi realizado por discentes pré-selecionados, capacitados para desempenhar as funções sob orientação dos pesquisadores.

Para inclusão no projeto era necessário que a adolescente residisse na área urbana da cidade de São Carlos/SP e que os pais e/ou responsáveis assinarem um termo autorizando. Encontraram nas visitas realidades que necessitavam de fato da intervenção de profissionais de saúde, como episiorrafia infeccionada e/ou com descência, bebês com letargia, secreção fétida no umbigo, entre outros, revelando a contribuição do projeto para a sociedade e aos discentes em sua formação profissional.

Através deste é evidenciado a importância da puericultura para o desenvolvimento de uma criança saudável, principalmente no contexto de vulnerabilidade que o nascimento por uma mãe adolescente proporciona. 
Melo; Coelho (2011) avaliaram o cuidado ofertado a grávidas adolescentes na Atenção Básica ( $A B)$, em uma cidade do interior da Bahia, através da observação da atuação e de entrevistas aos profissionais selecionados, sendo estes; duas enfermeiras, um médico e cinco Agentes Comunitários de Saúde (ACS), entre 2006 e 2007.

Segundo os autores é necessário que haja um acolhimento as gravidas adolescentes pelos profissionais de saúde, proporcionando as pacientes uma escuta sensível, que valorize o contexto destas. Fato que não se concretizou nas consultas pré-natal de enfermagem, pois os discursos revelaram uma abordagem coerente, mas que não aconteciam na prática.

$\mathrm{Na}$ observação da consulta médica e na entrevista ao profissional é evidenciada a fragmentação do cuidado, cabendo a enfermeira a responsabilidade dos atendimentos e quando ocorre a consulta pelo profissional médico ela é extremamente técnica. A forma de atenção ofertada pelos ACS às adolescentes não respeita a singularidade, desencadeando um distanciamento entre as jovens e o serviço de saúde.

"Considerada incapaz de construir e assumir responsabilidades, a grávida adolescente é colocada como espectadora no cenário do cuidado à saúde, negandose a oportunidade de se tornar sujeito ativo nesse processo" (MELO; COELHO, 2011). Revelando assim uma necessidade urgente de mudanças no atendimento a esse público.

Ainda com o intuito de examinar 0 atendimento ofertado as gestantes adolescentes o artigo de Vilarinho; Nogueira; Nagahama (2016) visa obter uma avaliação da qualidade através da análise de 174 prontuários e de entrevistas com 44 adolescentes que realizaram o pré-natal nas unidades de saúde no município e deram a luz numa maternidade de Teresina, Piauí, entre 01 de janeiro a 31 de dezembro de 2008.

De acordo com os prontuários da amostra em 13,2\% das gestantes o número de consultas pré-natal realizadas foi de 1 a 3, 47,7\% obtiveram de 4 a 6 e $39,1 \%$ de 7 a 10, é importante frisar que pode haver uma relação direta do número de consultas 
com o início do pré-natal, quanto mais cedo começar provavelmente maior será o número de consultas realizadas.

Quanto a este início do pré-natal, em 22,7\% das entrevistadas aconteceu tardiamente, com mais de 17 semanas de gestação, perdendo toda a atuação em saúde do primeiro trimestre, período em que há um maior risco para abortamento. Revelando então a necessidade de uma captação precoce, que proporcione acolhimento e criação de vínculo.

$\mathrm{Na}$ realização do exame Venereal Disease Research Laboratory (VDRL), para detecção da sífilis, 21,8\% não tinham registro em prontuário e 3,4\% não realizaram. Sobre o teste anti-HIV apenas $69 \%$ obtiveram o resultado registrado em prontuário, fato que requer uma mudança urgente, pois o desconhecimento sobre este resultado põe em risco a vida da adolescente, do bebê e dos profissionais de saúde que prestam o cuidado a paciente durante o trabalho de parto.

Os autores supõem que as adolescentes estão mais vulneráveis a estas infecções devido a precocidade do início da vida sexual e da instabilidade no uso de medidas preventivas, realçando assim a importância destes exames para esse público entes do nascimento.

A respeito dos exames de rotina, tipagem sanguínea, glicemia de jejum, urina tipo I, hemoglobina e hematócrito, todas as gestantes conseguiram realizar, em contrapartida apenas 18,2\% conseguiram mais de duas ultrassonografias, 50\% relataram que obtiveram duas e $31,8 \%$ apenas uma.

Sobre a vacinação antitetânica, segundo o registro em prontuário, apenas 8,6\% estavam imunizadas, $16,7 \%$ receberam a dose de reforço e 35,6\% três doses, para os autores esse baixo índice de imunização está relacionado com o fato de as adolescentes não terem o hábito de buscar a imunização nos serviços de saúde.

Quanto a orientação sobre a amamentação, $88,6 \%$ das entrevistadas indicaram que receberam durante as consultas, e que o exame das mamas também foi realizado em 
$75 \%$ delas, revelando que a equipe de saúde conseguiu ter uma boa desenvoltura quanto a este assunto.

Com relação à informação sobre o tipo de parto, a maioria das adolescentes $(81,8 \%)$ não foi orientada sobre as vantagens $e$ desvantagens dos tipos de parto. O pré-natal é o momento mais apropriado para a preparação e orientação para o parto. Além disso, no contexto de saúde sexual e reprodutiva, a escolha do tipo de parto constitui um dos principais direitos reprodutivos das mulheres (VILARINHO; NOGUEIRA; NAGAHAMA, 2016).

$\mathrm{Na}$ atenção puerperal, 52,3\% das entrevistadas não retornaram a UBS até o $42^{\circ}$ dia pós-parto e/ou não houve visita domiciliar na 1 a semana pós-parto, e apenas $70,5 \%$ foram orientadas sobre métodos anticoncepcionais nesse período, algo que precisa ser revisto pela equipe de saúde para que possam atuar de forma eficaz, prevenindo uma nova gestação e atuando sobre as intercorrências provenientes dessa fase de adaptação.

Ainda no contexto da Atenção Básica, o artigo de Buendgens; Zampieri (2012) foi desenvolvido em uma UBS do sul do Brasil, com o objetivo de conhecer como ocorre o atendimento da equipe de saúde e suas percepções sobre as adolescentes grávidas, através de entrevistas com 10 profissionais de saúde, sendo estes médicos e enfermeiros que realizaram consultas de pré-natal, durante o período de agosto a outubro de 2010.

Em relação a percepção dos profissionais sobre a gravidez na adolescência, os entrevistados apontaram que esse evento constitui um problema de saúde pública e que aumenta a possibilidade de "anemia, prematuridade, mortalidade neonatal e materna, ameaças de aborto, infecções, doença hipertensiva, parto prolongado, desnutrição, baixo peso dos recém-nascidos," entre outras complicações, colocando em risco a vida da adolescente e do bebê.

Para eles, a gravidez nessa faixa etária é um problema social, proveniente da falta de estrutura familiar e social, dos incentivos a sexualidade presentes na mídia e do tempo ocioso que a maioria dos adolescentes passam. E que a gestação traz como consequência a continuidade do ciclo da pobreza, e em alguns casos abandono pelo 
companheiro, família e amigos. Sendo essencial que os profissionais conheçam o contexto em que a adolescente está incluída, como também o significado que a gravidez tem para ela.

Sobre as mudanças sociais advindas da gravidez, os relatos citaram o afastamento do ciclo de amigos e o abandono escolar, por vergonha e/ou receio do julgamento dos professores e colegas, resultando em menores oportunidades de vida e de profissão, diminuindo a renda familiar e aumentando a dependência da família.

Nas mudanças físicas, citaram o aumento de peso e as estrias como principais alterações corporais, que as adolescentes desejavam ter de volta o corpo que tinham antes da gestação, o que gerava sofrimento e diminuição da autoestima, e que em algumas adolescentes a gravidez pode comprometer o desenvolvimento corporal normal para a idade.

Quanto as mudanças emocionais apresentaram que há uma ambivalência de sentimentos diante da gravidez, indo da indiferença, negação até a aceitação, medos em função do parto, de não saber cuidar do bebê e de como amamentar, provocando angústia, sofrimento e depressões, se considerando em uma situação de crise. Mas que tendem a serem amenizadas quando elas têm uma rede de apoio, principalmente do companheiro e dos familiares.

A respeito de suas atuações com a adolescente grávida, os profissionais relataram não haver equidade no atendimento prestado a esse grupo, sendo integral e de acordo com a demanda como nas demais, concentrando-se mais no físico do que no emocional e social. Justificando isto no fato das gestantes serem tímidas, de não haver espaços para interação e que nem sempre é possível dispor tempo para atender as necessidades das adolescentes, também relataram dificuldade para a formação de vínculo e de uma relação de confiança com elas.

Para os autores

A adolescente precisa ser vista em sua integralidade, com enfoque na saúde mental e emocional, e devem ser oportunizados espaços para o 
diálogo, compartilhamento de suas dificuldades e medos, conhecimento dos seus direitos e fortalecimento de suas potencialidades para fazer escolhas e repensar artigos projetos e sonhos (BUENDGENS; ZAMPIERI, 2012).

O artigo de Enderle et al. (2012) aborda a qualidade de atenção ao parto sob o olhar das adolescentes, através de entrevistas individuais com 269 pacientes, de idade entre 10 e 19 anos, que deram a luz no Hospital Universitário do Rio Grande, RS, entre julho de 2008 e outubro de 2009.

Para analisar a qualidade do atendimento ofertado, os autores apontaram as condutas e rotinas utilizadas no centro obstétrico local e compararam com as práticas indicadas a prestação de cuidado perinatal da OMS, que também são sugeridas pelo Ministério da Saúde (MS).

Os resultados obtidos revelaram um desconhecimento das adolescentes sobre os direitos da parturiente, tendo em vista que $94,4 \%$ das entrevistadas não tiveram acompanhantes no centro obstétrico e 93,3\% delas afirmaram não conhecer esses direitos.

Sobre a assistência prestada pela equipe, apenas 37,3\% delas receberam informações e orientações sobre o processo do trabalho de parto, o desconhecimento do quê e como vai acontecer pode gerar medo e ansiedade na maioria. Ainda sobre a assistência, 39,0\% das parturientes entrevistadas tomaram banho para obter relaxamento e higienização, favorecendo no alívio e controle da dor, técnica que deveria ter sido utilizada pela maioria.

Outro achado relevante na pesquisa é o alto índice de episiotomia, essa técnica foi realizada em 89,6\%, mas que é indicada apenas em 10 a 15\% dos casos, da mesma forma a restrição hídrica e alimentar que $87,4 \%$ das entrevistadas passou, embora seja rotina na instituição, é desnecessária, pois a maioria é primigesta, podendo levar em média 12 horas para concluir o trabalho de parto, e a restrição por este longo período de tempo além de gerar desconforto, reduz os níveis de glicemia, dificultando o processo. 
A respeito dos toques vaginais, para verificação da evolução do trabalho de parto, foram realizados em $69,4 \%$ das adolescentes por mais de um examinador, fato que gera desconforto, mas que pode ser justificado por se tratar de um hospital universitário e ter a presença contínua de acadêmicos. No entanto, $84 \%$ delas afirmaram ter sua intimidade e privacidade respeitadas.

Sobre o bebê, $89 \%$ relatou que amamentou na primeira hora de vida e $64,3 \%$ diz ter tido um contato precoce com o filho. Embora seja um número alto, para os autores ainda se faz necessário a adoção de estratégias de incentivo, no intuito de alcançar a totalidade.

De acordo com eles "receber atenção adequada no momento do parto pode ser um passo decisivo e importantíssimo para amenizar os agravos que podem advir de uma gravidez precoce" (ENDERLE et al., 2012).

Já o artigo de Gurgel et al. (2010) analisa, através da técnica de grupo focal, as práticas de 8 enfermeiros da Estratégia de Saúde da Família (ESF) em Fortaleza, Ceará, direcionadas à prevenção da gravidez na adolescência, no período de março e abril de 2008.

A análise revelou que 0 trabalho com grupos de adolescentes, ligados ao planejamento familiar, se faz presente no cotidiano de alguns dos enfermeiros, e para os que não conseguem desenvolver esse tipo de atividade em grupo há dificuldades organizacionais e estruturais que os impedem.

Segundo os autores a promoção de atividades com grupos de adolescentes facilita o debate, ampliando a compreensão de assuntos ligado a saúde, como também permite a reflexão sobre questões ligada a família e sociedade. Fato que aumenta o vínculo dos adolescentes com a equipe e torna possível que os participantes se tornem disseminadores de boas práticas em saúde.

Quando a educação em saúde é trabalhada no modelo convencional, na forma de palestras, apenas transmitindo conhecimento, os enfermeiros consideraram ineficaz e relataram evasão dos adolescentes presentes. Já a metodologia apresentada como 
tratamento de choque utilizada por alguns, onde há exposição de imagens fortes, com o intuito de chamar atenção para patologias, não é indicado, por não contribuir para a aprendizagem e afastar os jovens da ESF.

De acordo com os autores "as ações de Educação em Saúde devem ser instigantes, criativas, motivadoras e inovadoras, capazes de estimular o adolescente a participar do processo educativo. Devem contar com todas as opções e recursos disponíveis na comunidade" (GURGEL et al., 2010). Como citado no artigo, temos o exemplo de uma entrevistada que utiliza a rádio da escola local para propagar informações em saúde a esse público.

É essencial que o enfermeiro promova ações interdisciplinares de educação sexual, tanto na consulta de enfermagem, de forma individual, quanto coletivamente nos grupos, sempre valorizando os conhecimentos que os jovens já possuem e entendendo o contexto em que eles estão inseridos.

\section{CONSIDERAÇÕES FINAIS}

A adolescência é um período marcado por grandes mudanças e alta vulnerabilidade, principalmente nos jovens inseridos em famílias mais pobres. Nesse contexto o evento de uma gravidez pode gerar complicações para a saúde da adolescente, como para a do bebê, dificuldades financeiras e contribuir para a continuidade do ciclo de pobreza, já que as jovens tendem a sair da escola, diminuindo as oportunidades de trabalho.

Conforme citado nos artigos analisados, durante o período de pré-natal e puerpério o enfermeiro se apresentou como principal elo para promoção da saúde, suas atitudes são capazes de despertar um sentimento de apoio, através de conselhos, informações, demonstrações de carinho e preocupação, marcando a vivência da maternidade como algo positivo para as adolescentes, tanto no ambiente hospitalar quanto na atenção básica. 
É essencial que durante $O$ atendimento elas se sintam acolhidas, que sejam orientadas quanto a seus direitos, que os profissionais da saúde atuem com empatia e tenham abertura para diálogos, não concentrando-se apenas no físico, mas abordando também o campo das emoções. As jovens devem ser vistas como protagonistas durante todo o processo gestacional e dos cuidados com o bebê, não apenas como sujeito passivo á intervenções.

Em relação as ações para prevenção da gravidez é importante ressaltar que a atuação com grupos de jovens se apresentou muito útil em algumas UBS's, mas que é necessário ampliar essa atuação, através de atividades inovadoras, criativas, capazes de atrair os jovens, tanto na Unidade Básica, como nas escolas e nas mídias sociais, envolvendo esse público em atividades para promoção a saúde.

Embora os artigos analisados não abordem sobre a prevenção da gravidez na adolescência no ambiente escolar, requerendo uma pesquisa mais ampliada para tal, os resultados encontrados são de igual relevância e apontam para a necessidade de melhorias no atendimento ofertado pelos enfermeiros a esse público, tanto no ambiente hospitalar quanto na atenção básica.

\section{REFERÊNCIAS BIBLIOGRÁFICAS}

BERETTA, M. I. R. et. al. A construção de um projeto na maternidade adolescente: relato de experiência. Ver. Esc. Enferm. USP, Abr 2011, vol 45, no. 2, p.533-536.

BRAGA, I. F. et. al. Percepções de adolescentes sobre o apoio social na maternidade no contexto da atenção primária. Esc Anna Nery, Set 2014, vol 18, no. 3, p. 448-455.

BRASIL. MINISTERIO DA SAÚDE. Nascim p/ocorrênc por idade da mãe segundo Unidade da Federação. Período: 2017. Sistema de Informações sobre Nascidos Vivos SINASC. Disponível em 
tabnet.datasus.gov.br/cgi/tabcgi.exe?sinasc/cnv/nvuf.def . Ultimo acesso em : $13 / 09 / 2019$

BRASIL. MINISTÉRIO DA SAÚDE. Política Nacional de Atenção Básica. Departamento de Atenção Básica. - Brasília: Ministério da Saúde, 2012.

BUENDGENS, B.B. ZAMPIERI, M. F. M. A adolescente grávida na percepção dos médicos e enfermeiros da atenção básica. Esc. Anna Nery, Mar 2012, vol 16, no.1, p.64-72.

ENDERLE, C. F. et. al. Avaliação da atenção ao parto por adolescentes em um hospital universitário. Ver. Bras. Saude Mater. Infant., Dez 2012, vol.12, no.4, p.383394.

FERNANDES, R.A.Q. NARCHI, N.Z. Enfermagem e saúde da mulher. 2 ed. Barueri, SP: Manole, 2013.

GURGEL, M. G. I. et. al. Desenvolvimento de habilidades: estratégia de promoção da saúde e prevenção da gravidez na adolescência. Ver. Gaúcha Enferm. (Online), Dez 2010, vol.31, no.4, p. 640-646.

MELO, M. C. P. COELHO, E. A. C. Integralidade e cuidado a grávidas adolescentes na Atenção Básica. Ciênc. saúde coletiva. Maio 2011, vol.16, no.5, p. 2549-2558.

VILARINHO, L. M. NOGUEIRA, L. T. NAGAHAMA, E. E. I. Avaliação da qualidade da atenção à saúde de adolescentes no pré-natal e puerpério. Esc. Anna Nery, Jun 2012, vol.16, no.2, p. 312-319.

SOCIEDADE BRASILEIRA DE PEDIATRIA. Consulta do adolescente: abordagem clínica, orientações éticas e legais como instrumentos ao pediatra. Manual de Orientação. Departamento Científico de Adolescência. no.10, Janeiro de 2019. 
SOCIEDADE BRASILEIRA DE PEDIATRIA. Prevenção da gravidez na adolescência. Guia Prático de Atualização. Departamento Científico de Adolescência. No.11, Janeiro de 2019.

Enviado: Abril, 2020.

Aprovado: Outubro, 2020. 